\title{
In What Way Interactions amongst Social Entities Can be Integrated into Computations as Basis for Unravelling Complications
}

\author{
Khalid A. Fakeeh, PhD \\ King Abdullaziz University \\ Jeddah, Saudi Arabia
}

\begin{abstract}
A social network is a network of relationships and interactions among social entities such as individuals, organizations, and groups. Social simulations are capable of representing social systems and coupled socio-techno-natural systems in ways that other methodological approaches are not. Computer code in a well-chosen programming language or simulation system. The pivotal role that agent interaction plays in determining social patterns has been investigated by two leading sociologists, James S. Coleman and Raymond Bourdon. In the 1960s, Coleman significantly contributed to developing mathematical sociology, but also worked to extend equationbased models through computer simulation could motivate and agent-based turn in sociology. This approach would contribute to shifting away from social systems tradition and their large-scale problems of social speculation to problems which can be studied by systematic research. The purpose of this paper is to discuss the challenges facing social entities, and how social objects can be incorporated into computations as the basis for solving difficulties.
\end{abstract}

\section{Keywords}

Social Computing, Web-technology, Problems, Computation

\section{INTRODUCTION}

Social computing is a tool that is traditionally focused on connecting people to the internet are tested and exposed to the rigors of the marketplace; the gems have risen to the top. Taking these same concepts for social connectedness and applying them to business situations for deriving value can be a calculated risk for an organization to take. Social computing embraces the part where collective behavior and computer discipline interconnect. Human beings are communal beings who study, perform and work in social sceneries; as a consequence, people are enormously delicate to the conduct of those nearby them, and their character is eventually molded by their public setting. Social computing appliances upkeep one-to-many connections, and thus descriptions need to obscure single, group, and civil procedures. It is claimed that social discipline tactics have a great compact to give in comprehensive connections at each of these ranks. At the discrete height, communal science can clarify the influence of character and separate inequalities on the use of public computing and the effects of it on self-perception and sentiment. At the cluster rank, open science can assist in comprehending decision constructing of the group and cluster units. Additionally, social computing maintains the relations that are performed by an original significant group of persons or societies. Keeping in mind the challenges of social interactions in social entities, social computing can aid in solving such problems.

\section{LITERATURE REVIEW}

Structural inequality, whether global, regional or local is manifest in environmental problems much as it is in other areas of society. Where individuals or communities have limited life chances, the effects of environmental degradation exacerbate inequality through a greater impact on the resources needed to sustain life. The irony that an abundance of resources in developing regions might have adverse effects on the health and well-being of local populations is an important example of how the unequal distribution of wealth and power maintains the status quo both locally and internationally [1]. ICT expert Kimberley Springer (MSI '14, Social Computing Specialization) explains that social computing is a discipline in computer science that develops an algorithm that integrates social behaviour into computerized systems. It involves the development of software technology to analyze human behavior. The essence of this computation is the realization that there is a social basis for human behavior. MSI Springer explains that digital systems use socially produced data that support useful functionality to analyze human behavior. For Google page algorithms, information is directly provided given the number of searches that recursively point to them. Sources retrieved from the annals of computer history indicate that University of Illinoisbased PLATO computer system originated the social computing technology in 1973 in an archetypal live production environment. The campaign that kicked off in Urbana-Champaign involved social software applications for instant messaging, group message forums, and multi-user chat rooms. The email surfaced a year later along with News Report, the original online newspaper. Social computing was further popularized by James Surowiecki in his runaway success dubbed 'The Wisdom of Crowds,' which highlighted prediction markets, collaborative filtering, and online auctions. Structural inequality, whether global, regional or local is manifest in environmental problems much as it is in other areas of society. Where individuals or communities have limited life chances, the effects of environmental degradation exacerbate inequality through a greater impact on the resources needed to sustain life. The irony that an abundance of resources in developing regions might have negative effects on the health and well-being of local populations is an important example of how the unequal distribution of wealth and power maintains the status quo both locally and internationally [1]

\subsection{Applying Sociological Theory}

For a macro understanding of the environmental crisis, we can implement the two primary structural theories in modern sociology. Functionalism and Conflict Theory. They vary from each other in their methodologies to analyzing 
environmental problems. Both methods examine the groups and institutions that contribute to the shape of society and distribution of resources and interaction among social entities. Functional theory examines how society is structured and how the major social institutions function. It argues that social stability is created by balancing various contending forces such as social agencies and interest groups. According to this theory, social change occurs when social equilibrium fails due to dysfunction in the social system that requires society to correct the imbalance to maintain social continuity. Conflict theory, on the other hand, presumes that social equilibrium does not exist, and in fact, that society is constantly changing. The sources of change are founded in social inequalities between groups. This theory contends that inequality by its very nature leads to conflict brings about social change [2]. Some functionalists may argue that the environmental crisis serves a function by creating jobs to clean up toxic sites and developing opportunities for research and planning for cleaner energy. Alternatively, functionalists may argue that the current environmental crisis with depleting resources and global warming is dysfunctional to society. Re-establishing social equilibrium would require rethinking energy consumption, which then challenges individualistic values and requires a radically different approach that considers changes in every social institution. Retooing for clean industry and dramatically reducing dependency on fossil fuels requires immense changes in engineering and infrastructure that are costly and time-consuming. Halting climate [2], the change would demand that all the major social institutions adapt new ways to function to limit their environmental impact. There are already signs of change in our values, even among large corporations that promote their green image. Other instances that may appear as a social response to a dysfunctional environment include recycling, new laws demanding a reduction in carbon dioxide emissions in cars and factories and buy-local movements, among others.

\section{PROBLEM STATEMENT}

We cannot define the limits of a social system devoid of examining the relationships or the interactions among its individual members. Merely noting their similar attributes will not suffice; as Miller himself points out, Sherlock Holmes quite correctly assumed that all of the red-haired men in London did not constitute a system until we found traces of organized relationships among them. Since any social system implies both entities and relationships among them, there seems little point in differentiating between concrete systems; we cannot have a social system unless both sets of phenomena are present. The second set of problems raised by Miller's distinctions is epistemological. They garble the relationship between the empirical world about which we generalize, and the numerical, verbal or graphic symbols we use in articulating such generalizations.

\section{DATA ANALYSIS AND APPLICATION TO REAL LIFE SCENARIOS}

4.1. Social system examination signifies a technique for obtaining logical consequences in about nearly any group interrelations in which communal units are present. In SNA (Social Network Analysis) range, group subtleties are studied to recognize relations and interfaces among their associates. Beginning from these connections, it is likely to recognize social designs and to spot or suggest social structural variations that disclose how systems develop or should revolve. Besides, it is probable to find possible reasons and effects of a network alteration, forecasting, and regulating networks' progress. These structures are reliant on metrics which permit group possessions' documentation or portray individual impact on a precise group. To accomplish SNA, it is vital to outline actions that can be contrasted between performers or nets. Methods in SNA can be renowned as those that gauge the whole system and the ones that only evaluate a precise node. The social interacting examination can use in society to better comprehend the communal wealth, support corporations, and associations; calculate the degree of embedment of the practitioners in addition to their significance in the system; support information administration.

4.2. Sociologists consider the family as the basic social unit. As the primary social unit, the family is a key agent of socialization responsible for the emergence of personality traits, allocation of designed social roles and development of behavior through nurturance. Second marriage is a form of quasi-family model that departs from the first-time conventional union. It has become a household trend today as the rate of divorces keeps increasing. While developing the structural family theory, Salvador Minuchin studied human behavior extensively (Forbes 23). In his research, he found that human behavior is subjective; an individual's behavior is a function of their relations with others. In the family setting, spouses, children, and other kin have dynamic links, which are often complex. The complexity of family relations has been the center of academic inquiry for years as scholars seek to establish what causes the dysfunctions. Dysfunctions within the family have continually caused family disintegration and the subsequent formation of new households. Minuchin's family structural theory deals primarily with the issue of second marriages focusing on how to make new family members coexist harmoniously. Other family theorists concur that the formation of new families based on second marriages is often marred by conflict in the form of internal fighting. Using Minuchin's family structural theory, scholars have suggested various ways of making second marriages work. The first and most important thing is a compromise; all family members must learn to compromise for families to function. Understanding comes in the form of being sensitive to other people as well as being reasonable and pragmatic. As family members interact, they create what Minuchin calls a 'matrix of identity.' The model of identity, in turn, helps individuals understand and appreciate fellow family members by respecting their identity. For instance, once a woman remarries a man who already has children from a past marriage, it is always important to comply with the status of the new family members. Understanding the character of people from both sides is the first step towards building meaningful family relations. This helps in national role designation. Every family member ought to understand their internal roles perfectly to avoid conflict of duties. A recent survey conducted under the auspices of Gallup International indicates that the issue of domestic roles is one of the primary reasons why families disintegrate. For instance, the father is often the designated breadwinner in a conventional family setting; this may change upon the birth of second marriages depending on the arrangements the new family makes. It is important as well to be gender-sensitive to avoid the perceived inferiority or superiority of either gender. Echoing the same, Professor Erickson McKinley of Columbia University notes that divorce and subsequent remarriage is a prevailing trend in the modern society. He espouses that there 
are certain complexities that mark the disintegration of one family and the emergence of another. There are a set of integrated values that are deemed appropriate in such situations. These values include compassion, love, responsibility to protect, duty to care, and mutual respect. By integrating these values, second marriages can be able to function well. The most important thing is that new families can learn from the dysfunctions that caused the disintegration of the initial family settings.

\section{APPROACH OR METHODOLOGY AND DATA ANALYSIS}

5.1. Methodology is a cognitive discipline in the knowledgeproduction process devoted to a systematic and logical study of sets of correct and incorrect techniques, methods, rules, procedures and principles of reasoning that may be used within a given paradigm over the epistemological space for knowledge search in various education sectors as may be needed by nature of the specific subject matters to deal with the possibility space, the probability space and the space of epistemic actual [3]. The method of research includes both qualitative and quantitative analysis. I also used both primary and secondary methods and study. Among the methods include a questionnaire, use of books, use witnesses, and study of the environment. I did an analysis of legal systems through automated information extraction, complexity modeling, and social network analysis.

5.2. Social network analysis is a vital technique in modern sociology. It is the study of patterns of relations, not just relationships between pairs; SNA differs from traditional data analysis in a way that its primary aspect of the report is attributes of links between objects rather than only object attributes. Hence, it offers quantitative measures to study the quantitative nature of relationships among individuals within a social group. Social networks are modeled as a graph of linked individuals called as a social diagram or social network diagram. SNA involves applying various analytical graph algorithms to extract interesting patterns of interaction and knowledge from the given instance of social charts. The social networks are analyzed in three diverse ways. For starters, it involves analyzing all objects of a population under consideration and connections among them. Secondly, by analyzing ego-centric networks which are created by extracting only interested objects and interactions among them. In this case, it is required to understand personal community network and its effect on involved individuals [4]. The modeling and scrutiny of multifaceted interaction data is a vigorous research topic in the social science and statistical physics communities. Real world systems such as the internet, socio-economic interactions, and biological networks have been extensive studies from an experimental viewpoint, and this has led to the development of a variety of models to understand their topological properties and evolution. In particular, technological networks, social interaction graphs, and graph abstractions in biology are shown to exhibit common structural features like flat graph diameter, skewed vertex degree distribution, self-similarity and dense subgraphs. Analogous to the small world phenomenon, these real world data sets are broadly referred to and modeled as small-world networks [5]. Among the many methods, social media was used to analyze networking data. Focusing on Twitter, it appears that it is the most powerful platform when it comes to economic growth through networking. Such analysis on Twitter data ought to utilize digital means acknowledging how platform affordances impact the growth of networks such analysis is specifically well-suited to social scientists [5]. The relative openness of Twitter in contrast to other platforms has formed an extraordinary wealth of social media data and stimulated rapid and vital innovation in computational tools to collect, evaluate and visualize this information in the digital humanities and social sciences. The table/figure below indicates the use of social media platforms and their results after analysis.

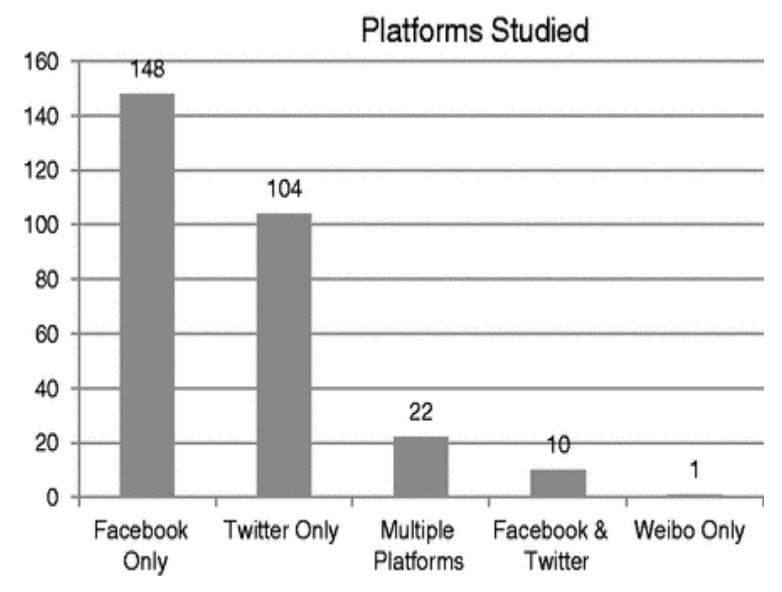

Figure 1: Usage (\%) of Different Platforms

\section{CONCLUSION}

In this paper we evaluated the problems encountered within social entities, the importance of social computing and how interactions amongst social entities can be incorporated into computations as a basis for resolving glitches. Problems within social entities are common and can be solved. The use of social computing will not only enhance vast networks among people but will turn the world into a better living place.

\section{REFERENCES}

[1] Göbel, S, TIDSE 2006, R. Malkewitz, \& I. Iurgel. "Technologies for interactive digital storytelling and entertainment: Third international conference, TIDSE 2006, Darmstadt, Germany, December 4-6, 2006 : proceedings". Berlin: Springer, 2006.

[2] J. Top of Form Elen, "Handling complexity in learning environments: Theory and research. Amsterdam [u.a.: Elsevier", 2006. Top of Form

[3] M.M. Fischer, "Spatial analysis and geo-computation: Selected essays". Berlin: Springer, 2006

[4] M. L, Top of Form Gavrilova. "Generalized Voronoi diagram: A geometry-based approach to computational intelligence." Berlin: Springer, 2008.

[5] M. M, Top of Form In Cruz-Cunha, F. In Moreira, \& J. In Varajao. "Handbook of research on enterprise 2.0: Technological, social, and organizational dimensions", 2014. 